\title{
Design and Realization of Industrial Control System Serial Communication Based onVB and Mitsubishi Programming Interface Protocol
}

\author{
Sun Xiao-hua \\ Digital Transmit Department \\ HeBei Software Institute \\ HeBei Baoding, China \\ 707412641@qq.com
}

\author{
Wang Fu-shun \\ College of Information Science \& Technology \\ Hebei Agricultural University \\ HeBei Baoding, China \\ wangfushun@yahoo.cn
}

\begin{abstract}
By analyzing the current serial communication, used in a popular monitor system composed of PC, GOT and PLC, introduced a new design and implementation plan of serial communication based on $\mathrm{Vb}$ and Programming Interface Protocol. System achieved the efficient, stable and reliable communication under simple attachment without any mitsubishi's special communication board. The communication applied mitsubishi FX series PLC programming interface protocol, no longer need to set up the D8120, simplified the PLC program; Under this protocol and based on VB6.0 development environment, write the serial communication program adapt to FX full series, the program make the PC can read/write the PLC soft equipment and forced ON/OFF directly. In the actual application, No matter accuracy and stability of communication were to achieve requirements of industrial control system.
\end{abstract}

Keywords- Serial Communication; Programming Interface Protocol; VB6.0; PLC

\section{INTRODUCTION}

Along with the rapid development of industrial control technology and computer technology, the programmable logic controller got more and more widely used with its high reliability, modular structure, simple programming, etc. Some small and medium-sized automatic control equipment consists of PC, touch screens and PLC was very common model. Outside the basic function of programming to touch screen and PLC, PC remote monitor the whole control process and send/receive data packet etc maily. Through the touch screen, users could set system parameter and monitor in line directly. PLC implemented descending control instruction from PC and touch screen, at the same time upward transmit data. To realize the system monitoring, communication was the premise, namely the mutual communication of PC, touch screen and PLC. System introduced a new type of communication scheme, achieved the efficient, stable and reliable communication under simple attachment, Under Mitsubishi Programming Interface Protocol and based on VB6.0 MSComm control, realized the perfect communication of PC through F940GOT to FX2N series PLC.

\section{HARDWARE CONNECTION}

Fig1 said the connections of PC, PLCand touch screen F940GOT, GOT has two connectors as shown on the right side of figure 1. Among them, the (1) connector was used by PLC (RS422 connector), and the (2) connector was used by PC (RS232 connector). In the system, using PC's com1 port to conect with GOT's (2) connector through the RS232C connector directly, and GOT's (1) connector conect with PLC programming Interface through the RS422. In this connection mode, GOT and PC could be used at the same time, and PC could read PLC data pass through GOT directly, and not influenced by it ${ }^{[1]}$.

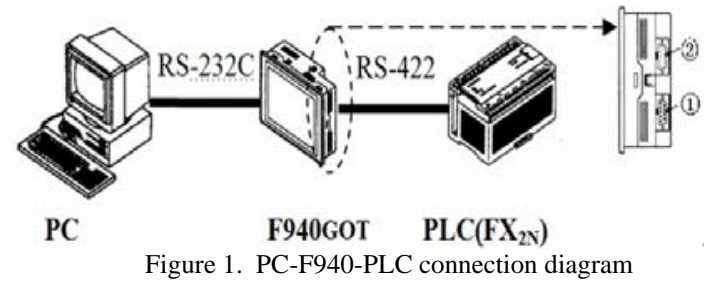

\section{SEAMLESS COMMUNICATION OF PC ,PLC AND GOT}

\section{A. Communication Settings of PC and F940GOT}

F940GOT picture production software must install in pc, and then pc could edit the picture and transmit or read the contents of GOT. In this system, installed the software of SW0PC - FXDU/WIN - C.As shown in Fig. 1, F940GOT has two interfaces, through the RS232C connector PC's serial port connect to GOT's (2) connector, GOT and PLC's programming interface linked together through the RS422 way. If PLC model was set in GOT's "action environment Settings” menu, so PLC, GOT and PC can transmit data each other and needn't to program PLC.namely, PC can program GOT and plc, and read or write data to them directly.

PC-F940GOT-FX2NPLC system, did not any special communication board, could realize presence control through touch screen. However, under this monitoring modle, it should be noted that PC's serial port communication parameters should be set to 9600, e, 7, 1 .

\section{B. Command code of FX-PLC and PC communication}

\section{TABLE I. COMMUNICATION COMMAND CODE}




\begin{tabular}{|c|c|c|c|c|}
\hline Instruction & Order & ASCII & Target soft element & Function \\
\hline Read & 0 & $30 \mathrm{H}$ & X Y M S T C D & Read state or data \\
\hline Write & 1 & $31 \mathrm{H}$ & X YMSTCD & Write data or"0","1" \\
\hline Set ON & 7 & $37 \mathrm{H}$ & X Y M S T C & Forced ON soft element \\
\hline Set OFF & 8 & $38 \mathrm{H}$ & X Y M S T C & Forced OFF soft element \\
\hline
\end{tabular}

Table I listed the command code used in serial communication. In the column of target soft element, $\mathrm{X}, \mathrm{Y}$, $\mathrm{M}, \mathrm{S}, \mathrm{T}, \mathrm{C}$ and $\mathrm{D}$ referred to input relay, output relay, auxiliary relay, state components, timer, counter and data register separately ${ }^{[2]}$.

\section{Control character of FX-PLC and PC communication}

Table II listed the serial communication control characters used by PC and FX series PLC. Mitsubishi FX series PLC programming port communication protocol used RS-232C standard, and PC's serial port communication parameters should be set to 9600 , e, 7, 1. The control characters must be converted to Hex ASCII code in the process of Communication. In VB program, if using characters in talbe II to take place of ASCCII code, the list of " Application in VB " in in table II should be programed.

TABLE II. COMMUNICATION CONTROL CHARACTER

\begin{tabular}{cccc}
\hline Character & ASCII & Application in VB & Function \\
\hline ENQ & $05 \mathrm{H}$ & ENQ\$=Chr\$(5) & PC send request to PLC \\
ACK & $06 \mathrm{H}$ & ACK\$=Chr\$(6) & PLC's Correct response \\
NAK & $15 \mathrm{H}$ & NAK\$=Chr\$(21) & PLC's Error response \\
STX & $02 \mathrm{H}$ & STX\$=Chr\$(2) & Message start mark \\
ETX & $03 \mathrm{H}$ & ETX\$=Chr\$(3) & Message end mark \\
\hline
\end{tabular}

The data-transport format of each ASCII character as shown in figure 3 shows. Among those bits, One bit as start bit, seven bits as data bits (low in the front, and high in the later), one bit as even check bit and one bit as stop bit, baud rate for 9600 BPS.

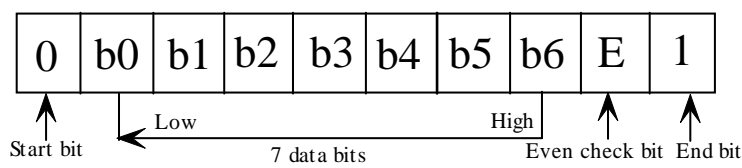

Figure2. Single character data send format

\section{Message analysis through example}

Ask and answer responder model used in PC and PLC communication. In the communication process, the request would be organized to sent again if the transmission error. The specific process was described in below, PC send request (ENQ) to PLC, then wait for PLC response, if the PLC gave correct response (ACK), then could send command message, and after received command message PLC would transfer response message to PC. However, If the PLC gave error response(NAK) then the request would be organized to sent again.The following was using example to analyze the message.
1) Read D data section of PLC

PC send message to PLC, its format as follows:

\begin{tabular}{|c|c|c|c|c|c|}
\hline Start & Order & First Address & Digit & End & Sum Check \\
\hline STX & CMD & ADDRESS & BYTES & ETX & SUMH,L \\
\hline
\end{tabular}

Above, STX for begin mark; CMD for order's ASC II code;ETX for end mark; SUM for the sum of all bytes from CMD to ETX and regardless of the overflow, it was composed of SUMH and SUML due to each byte hex number must be converted to ASCII code with two bytes. GROUP ADDRESS for the first address of PLC's operated unit, it could be calculated according to the mitsubishi FX series PLC communication manual and FX - 232 aw manual;BYTES for number of operated data byte began from the first address ${ }^{[3]}$.

The return message format from PLC as follows:

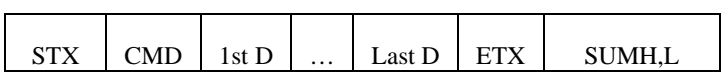

Example 1: Read 3584 from D123 section. Its address algorithm for ADDRESS $=123 * 2+1000 \mathrm{H}$, changed to Ascii code with $31 \mathrm{H}, 30 \mathrm{H}, 46 \mathrm{H}, 36 \mathrm{H}$.

PC send message to PLC as follows:

\begin{tabular}{|l|l|l|l|l|l|}
\hline $02 \mathrm{H}$ & $30 \mathrm{H}$ & $31 \mathrm{H}, 30 \mathrm{H}, 46 \mathrm{H}, 36 \mathrm{H}$ & $30 \mathrm{H}, 31 \mathrm{H}$ & $03 \mathrm{H}$ & $37 \mathrm{H}, 34 \mathrm{H}$ \\
\hline
\end{tabular}

PLC should respond correctly:

\begin{tabular}{|l|l|l|l|l|}
\hline $02 \mathrm{H}$ & $30 \mathrm{H}$ & $33 \mathrm{H}, 35 \mathrm{H}, 38 \mathrm{H}, 34 \mathrm{H}$ & $03 \mathrm{H}$ & $44 \mathrm{H}, 36 \mathrm{H}$ \\
\hline
\end{tabular}

2) Write data to D soft element of PLC

PC send message to PLC, its format as follows:

\begin{tabular}{|c|c|c|c|c|c|}
\hline Start & Order & First Address & Digit & \multicolumn{2}{|c|}{ Data } \\
\hline STX & CMD & ADDRESS & BYTES & 1ST D & 2ND D \\
\hline
\end{tabular}

Add...

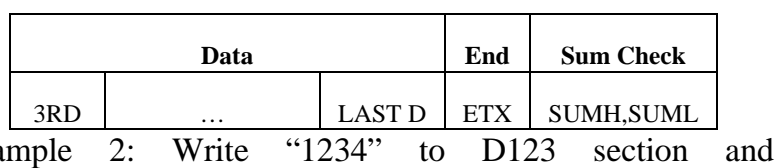
write“ABCD” to D124 section.

PC send message to PLC as follows:

\begin{tabular}{|c|c|c|c|}
\hline $02 \mathrm{H}$ & $31 \mathrm{H}$ & $31 \mathrm{H}, 30 \mathrm{H}, 46 \mathrm{H}, 36 \mathrm{H}$ & $30 \mathrm{H}, 34 \mathrm{H}$ \\
\hline
\end{tabular}

\begin{tabular}{|l|l|l|}
$33 \mathrm{H}, 34 \mathrm{H}, 31 \mathrm{H}, 32 \mathrm{H}, 43 \mathrm{H}, 44 \mathrm{H}, 41 \mathrm{H}, 42 \mathrm{H}$ & $03 \mathrm{H}$ & $34 \mathrm{H}, 39 \mathrm{H}$ \\
\hline
\end{tabular}

PLC's response cases as follows: if return with ACK $(06 \mathrm{H})$, indicate correct; but NAK $(15 \mathrm{H})$ for error.

3) Situation for bit element forced ON or OFF

\begin{tabular}{|c|c|c|c|c|c|}
\hline Commentary & Start & Order & Address & End & Sum Check \\
\hline Format & STX & CMD & ADDRESS & ETX & SUMH \\
\hline Force ON & $02 \mathrm{H}$ & $37 \mathrm{H}$ & address & $03 \mathrm{H}$ & sum \\
\hline Force OFF & $02 \mathrm{H}$ & $38 \mathrm{H}$ & address & $03 \mathrm{H}$ & sum \\
\hline
\end{tabular}




\section{USING VB6.0 TO REALIZE COMMUNICATION}

Regardless of the PLC's Stop or Run state, the communication program which was written with using VB6.0 MSComm control components, in addition to the constant value of PLC timer and counter and the data of file register, could use the communication command in the table 1 to read/write for all soft element and to set/reset for all switch. The soft element's address, which used in the programming, could be calculated out according to the FX232AW manual ${ }^{[4]}$.

\section{A. Profile for MSComm}

MSComm control transmited and received data through the serial port, provide serial communication function for the application. The control could set serial communication data to send and receive, and setted the message format and protocol of the serial port state and serial communication. Each communication control corresponding to a serial port, could design more communication control to access multiple communication port. MSComm control provided a series of standard communication properties and methods, which could build the connection of applications and serial port. The control shielded the bottom operation in communication process, programmers only needed to set up and monitor MSComm control's properties and events to achieve serial asynchronous communication ${ }^{[5]}$.

MSComm control's main attributes included: (1)CommPort : Set and return the communication port; (2) Settings: Set and return baud rate, parity, data bits and stop bits in the form of a string; (3)PortOpen: Set and return the state of communication port, and open and closing port; (4)Input: Return and delete character from the receive buffer; (5) Ouput : Write characters to transmission buffer; (6) InputLen: Set and return Input attribute from the number of characters which were read in the Input buffer, If setting to zero, it could read out all characters in the input buffer; (7) InbufferCount : Receive bytes in the buffer; (8) OutbufferCount: Output bytes in the buffer ${ }^{[6]}$.

System designed the VB application interface as what in figure 4 showed, serial communication program which used MSComm control generally design steps: (1) Set up communication object, communication port and other attributes; (2) Set the communication agreement; (3) Open communication port, and transmit data; (4) Close communication port. The following VB routine was conducted according to the above-mentioned steps ${ }^{[7]}$.

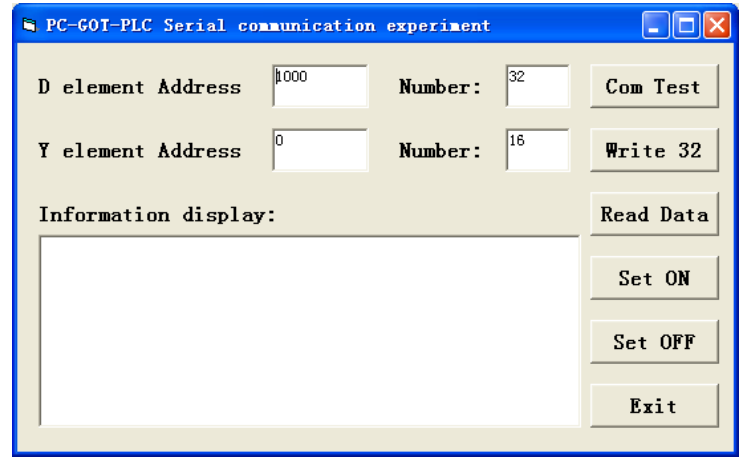

Figure3. VB application interface in experiment

B. Realizing communication in the VB6.0

1) $\mathrm{PC}$ communication port's initialization

MSComm1.CommPort $=1$

MSComm1.Settings = "9600,e,7,1"

MSComm1.InputLen $=0$

MSComm1.OutBufferCount $=0$

MSComm1.InBufferCount $=0$

MSComm1.PortOpen $=$ True

2) Writed the array data (1 to 32) to PLC's 32 units which began from D1000

Dim f1, f2, ff, fc, ff32 As String

Dim hxstr, setin As String

Dim $\mathrm{j}$ As Integer

$\mathrm{ff}=\operatorname{Hex}(1000 * 2+4096)$

$\mathrm{ff}=\operatorname{Right}(" 0000 "+\mathrm{ff}, 4)$

ff32 = "'"

For $\mathrm{j}=1$ To 32

fc = Right("0000" + Hex(data(j)), 4)

f1 $=$ Right(fc, 2)

$\mathrm{f} 2=\operatorname{Left}(\mathrm{fc}, 2)$

$\mathrm{ff} 32=\mathrm{ff} 32 \& \mathrm{f} 1 \& \mathrm{f} 2$

Next j

hxstr = "1" \& ff \& "40"

hxstr $=$ hxstr + ff32 + Chr\$(3)

MSComm1. OutBufferCount $=0$

MSComm1.InBufferCount $=0$

MSComm1.Output $=$ Chr $\$(2)+$ hxstr + SumChk(hxstr)

setin $=$ MSComm1.Input

If Asc(setin) $=6$ Then

End If

MsgBox " Write success!"

If Asc(setin) $=21$ Then

MsgBox " Write error !"

End If

3) Read content from the D1000 soft element area

Dim setin, hxstr, hxstr1 As String

Dim tim As Double

MSComm1.PortOpen=True

MSComm1.InBufferCount $=0$

MSComm1.OutBufferCount $=0$

MSComm1. RThreshold=11

hxstr=hxstr \&"0" \& Hex(1000*2+4096)\&"02"+Chr\$(3)

MSComm1.Output $=$ Chr $\$(2)+$ hxstr+SumChk(hxstr)

setin= MSComm1.Input

Textplc.Text=Val("\&H"+Mid(setin,4,2)+Mid(setin, 2, 2)) 


\section{CONCLUSION}

Using VB6.0 development platform, only through the simple hardware connection, System achieved communication of PC machine, touch screen and PLC with each other under the mitsubishi's PLC programming port agreement without any mitsubishi's special communication board. It not only simplified the PLC program, but also realized stable and reliable communication without setting up the PLC's D8120. In the small and medium-sized control system, Visual monitoring software, which was developed using VB, had friendly interface, pertinence and powerful function. Its economy and practicability had a strong advantage Compared with configuration software (INTOUCH, KINGVIEW, etc.) which had expensive price and complex programming.

The following projects funded the paper: Scientific research and development plan of Baoding support project (12ZN020).

\section{REFERENCES}

[1] Mitsubishi Company. MITSUBSHI-GOT.F940GOT-SWD Operation manual[M]. Shanghai : 2006.

[2] Mitsubishi Company. MITSUBSHI FX Communication manual[M]. Shanghai : 2001.

[3] Mitsubishi Company. MITSUBSHI.FX-232AW Interface power Communication manual[M]. Shanghai : 2001.

[4] Gong Zhong-hua. Mitsubishi FX PLC application technology [M] . Beijing: Press of People's Posts and Telecommunication, 2010.

[5] Yu Guo-liang,Meng De-xin.The communicaiton between VB and MITSUBISH FX series PLC with programming cable SC09[J]. Microcomputer Information, 2007 (3):51-52

[6] Zhang Cong-zhi.Serial Communication between Personal Computer and PLC[J]. .Machine Tool Electric Apparatus, 2005(3):37-39.

[7] Qiu Li-hua. Visual basic programming tutorial[M] . Beijing: Mechanical Industry Press, 2001. 